\title{
Development and validation of uncertainty neutron transport calculations at an industrial scale
}

\author{
Julien Gaillet ${ }^{1, *}$, Thomas Bonaccorsi ${ }^{1}$, Gilles Noguere ${ }^{2}$, and Guillaume Truchet $^{1}$ \\ ${ }^{1} \mathrm{CEA} / \mathrm{DEN} / \mathrm{DER} / \mathrm{SPRC} / \mathrm{LPN}, 13108$ Saint-Paul-Lez-Durance, France \\ ${ }^{2} \mathrm{CEA} / \mathrm{DEN} / \mathrm{DER} / \mathrm{SPRC} / \mathrm{LEPH}, 13108$ Saint-Paul-Lez-Durance, France
}

Received: 27 October 2017 / Received in final form: 14 February 2018 / Accepted: 14 May 2018

\begin{abstract}
Evaluating uncertainties on nuclear parameters such as reactivity is a major issue for conception of nuclear reactors. These uncertainties mainly come from the lack of knowledge on nuclear and technological data. Today, the common method used to propagate nuclear data uncertainties is Total Monte Carlo [1] but this method suffers from a long time calculation. Moreover, it requires as many calculations as uncertainties sought. An other method for the propagation of the nuclear data uncertainties consists in using the standard perturbation theory (SPT) to calculate reactivity sensitivity to the desire nuclear data. In such a method, sensitivities are combined with $a$ priori nuclear data covariance matrices such as the COMAC set developed by CEA. The goal of this work is to calculate sensitivites by SPT with the full core diffusion code CRONOS2 for propagation uncertainties at the core level. In this study, COMAC nuclear data uncertainties have been propagated on the BEAVRS benchmark using a two-step APOLLO2/CRONOS2 scheme, where APOLLO2 is the lattice code used to resolve Boltzmann equation within assemblies using a high number of energy groups, and CRONOS2 is the code resolving the 3D full core diffusion equation using only four energy groups. A module implementing the SPT already exists in the APOLLO2 code but computational cost would be too expensive in $3 \mathrm{D}$ on the whole core. Consequently, an equivalent procedure has been created in CRONOS2 code to allow full-core uncertainty propagation. The main interest of this procedure is to compute sensitivities on reactivity within a reduced turnaround time for a 3D modeled core, even after fuel depletion. In addition, it allows access to all sensitivites by isotope, reaction and energy group in a single calculation. Reactivity sensitivities calculated by this procedure with four energy groups are compared to reference sensitivities calculated by the iterated fission probability (IFP) method in Monte Carlo code. For the purpose of the tests, dedicated covariance matrix have been created by condensation from 49 to 4 groups of the COMAC matrix. In conclusion, sensitivities calculated by CRONOS2 agree with the sensitivities calculated by the IFP method, which validates the calculation procedure, allowing analysis to be done quickly. In addition, reactivity uncertainty calculated by this method is close to values found for this type of reactor.
\end{abstract}

\section{Introduction}

The studies of conception and safety as well as the exploitation of the reactor require simulation tools which have to be adaptative, reliable and able to predict fission chain reactions. These tools use nuclear and technological data to model core and physics. But these data contain uncertainties. The goal of this paper is to suggest a method able to propagate nuclear data uncertainties at industrial scale with short calculation time on a 3D modeled core and therefore to estimate uncertainty due to nuclear data on

\footnotetext{
* e-mail: julien.gaillet.jg@gmail.com
}

reactivity. This method relies on the sensitivities calculation due to nuclear data with the full core diffusion code CRONOS2. This work has been done on a two steps deterministic calculation scheme APOLLO2/CRONOS2 $[2,3]$ applied on the BEAVRS benchmark [4]. A CRONOS2 procedure calculating reactivity sensitivities to nuclear data by standard perturbation theory (SPT) [5-7] has been developed. Sensitivities are available by energy group and by reaction. The obtained results about sensitivities are compared to iterated fission probability (IFP) calculations [8-10]. These sensitivities will be used in uncertainties calculations on reactivity with condensed covariance matrix to determined uncertainty du to nuclear data on BEAVRS core. 


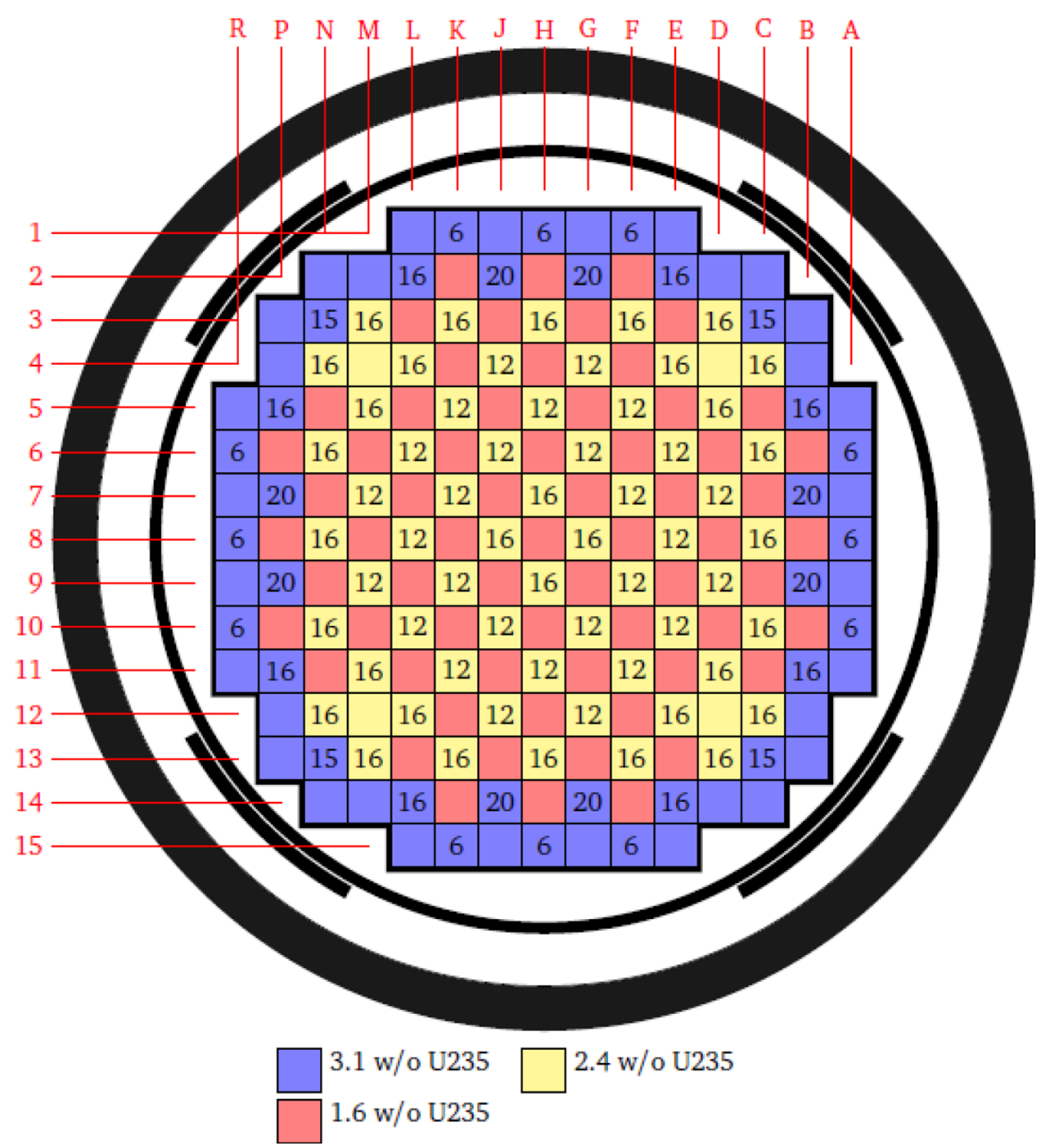

Fig. 1. Composition of BEAVRS core at cycle 1.

\section{The BEAVRS benchmark}

The Massachusetts Institute of Technology has proposed a benchmark of the BEAVRS reactor [4] based on two operational cycles to provide a highly-detailed PWR test case for the validation of high-fidelity core analysis methods. It is one of Beaver Valley plants reactor with a 931 MW electric power, located in Pennsylvania in the United States. The benchmark contains all the detailed material compositions and geometrical data for the major core constituents including the assemblies, baffle and the barrel. The core radius is about $2 \mathrm{~m}$ and its height is $4.5 \mathrm{~m}$. In hot zero power (HZP) conditions, the core temperature is $567 \mathrm{~K}$ with a 155 bar pressure. The core reactivity is controlled by boric acid contained in the water of the primary circuit and by burnable poison in pyrex pins. Pyrex is glass loaded with boron. It enables to balance reactivity in the beginning of life.

Cycle 1 and 2 differ by assemblies enrichment and by their number of burnable poison pins. In this paper, the cycle 1 data are used and are described in the following. The core is composed of 193 assemblies with three different enrichments in U235: 1.6\%, 2.4\% and 3.1\% (Fig. 1). Some assemblies contain pyrex pins inside guide tubes. The number of pyrex pins and their positions are shown in Figure 2. Each association between enrichment and the number of pyrex pins represents an assembly. So, there are nine different types of assembly. An assembly owns 289 pins, distributed into a $17 \times 17$ lattice (Figs. 3 and 4). Each pin can be a fuel pin or a guide tube. The guide tube can be empty (filled with water), or can contain pyrex pin, control rod in AIC (Argent, Indium, Cadmium) or instrumentation at the center of the lattice. The pin lattice pitch is $1.25984 \mathrm{~cm}$.

\section{Calculations scheme of BEAVRS}

The used calculation scheme in this paper is a two step APOLLO2/CRONOS2 scheme. Each assembly is modeled in 2D in APOLLO2 multi-group code resolving Boltzmann equation. Input data are nuclear data from JEFF-3.1.1 library [11] and technological data described assembly geometry and composition from BEAVRS benchmark. A first energetic condensation is done to 281 energy groups corresponding to the SHEM mesh [12]. It is the optimized mesh for self-shielding which is performed with Pij method. Then, a condensation to 49 energy groups is done to improve time calculation while maintaining a good accuracy. The flux 


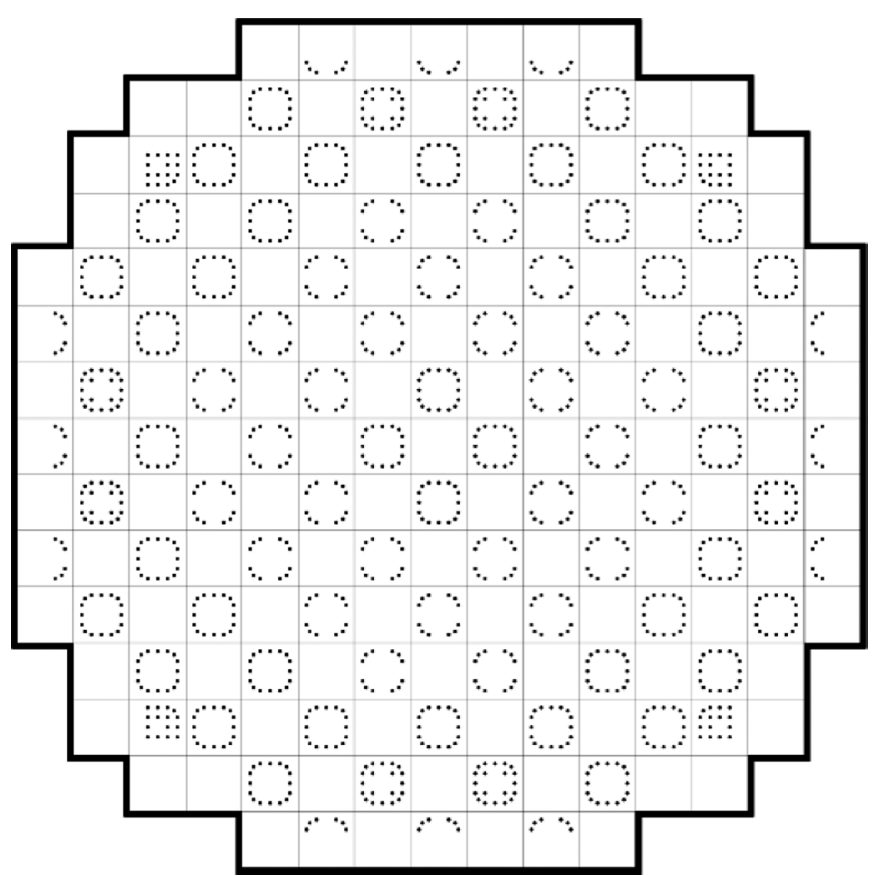

Fig. 2. Number and positions of pyrex pin at cycle 1 .

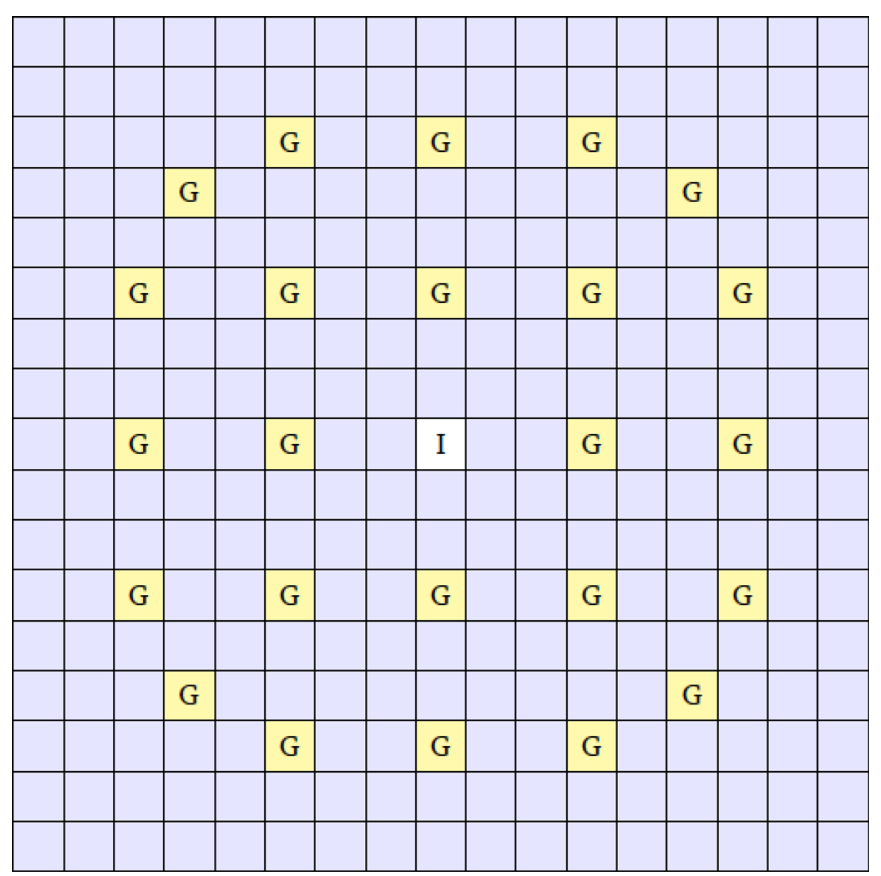

Fig. 3. Assembly without pyrex pin.

is calculated with a MOC method. Finally, self-shielding cross sections are condensed to 4 energy groups and are stored. Equivalent coefficients transport/diffusion allowing to preserve reactions rates between the two codes are also calculated. The second step of this calculation scheme is at core scale using CRONOS2 3D code. It uses stored cross sections computed for each assembly by APOLLO2 and it solves flux calculation in diffusion theory with 4 energy groups thanks to MINOS solver. Then, the

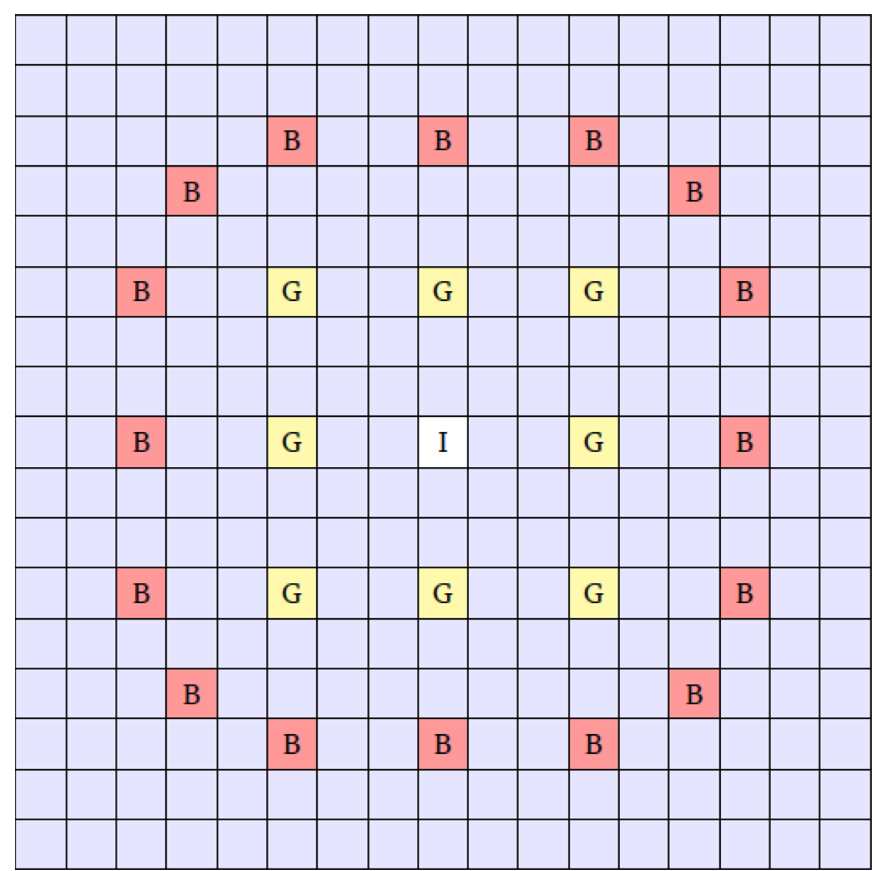

Fig. 4. Assembly with 16 pyrex pin.

CRONOS2 procedure developed for reactivity sensitivities calculation can be called. The sensitivity of parameter $k$ to cross section $\sigma$ in the energy group $g$ is given by:

$$
S\left(k, \sigma_{g}\right)=-\frac{\left\langle\varphi^{+},\left(A-\frac{F}{k}\right)_{\sigma_{g}} \varphi\right\rangle}{\left\langle\varphi^{+}, F \varphi\right\rangle} .
$$

In this equation, $\varphi^{+}$is the adjoint flux, $F$ is the neutron production operator and $A$ is the disappearance operator. In CRONOS2, the flux and the adjoint flux are calculated resolving diffusion equation but cross sections used in sensitivity equation are those which come from transport equation. Firstly, adjoint flux is calculated. Secondly, the procedure creates CRONOS2 structures which contain macroscopic cross sections of selected isotopes by reaction. These structures allow calculating production and disappearances operator for each isotope. Consequently, the scalar product with adjoint flux on phases space can be calculated and this enables to have production term. In addition, expression of sensitivity can be implemented and have to be breakdown by isotope, by reaction and by energy group. The split by energy group is made thanks to unitary sources which are equal to 1 in the wanted energy group and 0 in the other groups. Finally, the scalar product can be calculated and also the sensitivity on the reactivity for different isotopes and reactions. The low number of energy groups and the rough spatial mesh used in CRONOS2 code allow computing quickly sensitivities.

\section{Generation of four groups covariances matrix}

Four groups matrix have been created from condensation of 49 groups COMAC matrix. Condensation method relies 
Table 1. CRONOS2 sensitivities compared to IFP sensitivities coming from RMC code [8].

\begin{tabular}{llcrr}
\hline Isotope & Reaction & $\begin{array}{l}\text { CRONOS2 } \\
(\mathrm{pcm} / \%)\end{array}$ & $\begin{array}{r}\text { IFP (RMC) } \\
(\mathrm{pcm} / \%)\end{array}$ & $\begin{array}{r}\text { Difference IFP } \\
(\text { RMC)-CRONOS2 }(\%)\end{array}$ \\
\hline $\mathrm{U} 234$ & Capture & -1.42 & -1.50 & -5.33 \\
$\mathrm{U} 234$ & Fission & 0.04 & 0.04 & 0.00 \\
$\mathrm{U} 234$ & Neutron multiplicity & 0.07 & 0.07 & 0.00 \\
$\mathrm{U} 235$ & Capture & -97.26 & -99.60 & -2.35 \\
$\mathrm{U} 235$ & Fission & 460.09 & 441.00 & 4.33 \\
$\mathrm{U} 235$ & Neutron multiplicity & 931.92 & 927.00 & 0.53 \\
$\mathrm{U} 238$ & Capture & -259.32 & -217.00 & 19.50 \\
$\mathrm{U} 238$ & Fission & 44.15 & 46.10 & -4.23 \\
$\mathrm{U} 238$ & Neutron multiplicity & 68.64 & 72.90 & -5.84 \\
B10 & Capture & -163.91 & -153.00 & 7.13 \\
Zr90 & Capture & -0.94 & -1.78 & -47.19 \\
Zr91 & Capture & -6.63 & -5.06 & 31.03 \\
Zr92 & Capture & -1.95 & -1.82 & 7.14 \\
Zr94 & Capture & -0.70 & -0.64 & 9.37 \\
Zr96 & Capture & -0.87 & -0.67 & 29.85 \\
Sn112 & Capture & -0.02 & -0.02 & 0.00 \\
Sn115 & Capture & -0.03 & -0.06 & -50.00 \\
Sn116 & Capture & -0.13 & -0.13 & 0.00 \\
Sn117 & Capture & -0.14 & -0.12 & 16.67 \\
Sn118 & Capture & -0.11 & -0.09 & 22.22 \\
Sn119 & Capture & -0.09 & -0.09 & 0.00 \\
Sn120 & Capture & -0.04 & -0.04 & 0.00 \\
Sn124 & Capture & -0.03 & -0.03 & 0.00 \\
H2O & Capture & -65.80 & -64.50 & 2.02 \\
\hline & & & & \\
\hline
\end{tabular}

on the conservation of variance for a given isotope and for each group of four groups mesh. Variances are calculated by the following equation:

$$
V=S^{t} M S
$$

In this formulation, $M$ is the covariances matrix coming from database COMAC with 49 energy groups and $S$ is the vector of reactivity sensitivities to cross sections calculated at 49 energy groups by APOLLO2 on an assembly. So, the rate variance $a$ for a given isotope is:

$$
V_{a}=S_{a}^{t} M_{a a} S_{a} .
$$

And covariance between rates $a$ and $b$ is:

$$
V_{a b}=S_{a}^{t} M_{a b} S_{b}
$$

The variance of total rate for an isotope and which is conserved in this method is:

$$
V=V_{a}+V_{b}+2 V_{a b}
$$

In this case, uncertainties $I_{\sigma_{a}}^{g}$ and $I_{\sigma_{b}}^{g}$ in the group $g$ and correlations $r_{a b}^{g g^{\prime}}$ between cross sections $\sigma_{a}^{g}$ and $\sigma_{b}^{g^{\prime}}$ are given by:

$$
\begin{gathered}
I_{\sigma_{a}}^{g}=\sqrt{V_{a}^{g}}, \\
I_{\sigma_{b}}^{g}=\sqrt{V_{b}^{g}}, \\
r_{a b}^{g g^{\prime}}=\frac{V_{a b}^{g g^{\prime}}}{I_{\sigma_{a}}^{g} I_{\sigma_{b}}^{g^{\prime}}} .
\end{gathered}
$$

\section{Results on reactivity sensitivities for modeled 3D BEAVRS core}

The developed method in CRONOS2 code for the computation of sensitivities on reactivity due to nuclear data with four energy groups in diffusion theory has been tested on BEAVRS benchmark. The used configuration of the core is a critical configuration with all control rods output (ARO) with $975 \mathrm{ppm}$ of boron at HZP conditions. Reactivity sensitivity calculations on reactivity in CRONOS2 code have been compared to IFP reference calculations coming from RMC code [8] shown in Table 1 for each isotope and each reaction. Sentivities to scattering cross sections are not available for the moment in the CRONOS2 code and 
Table 2. CRONOS2 sensitivities compared to IFP sensitivities coming from TRIPOLI4 code [6].

\begin{tabular}{llrrr}
\hline Isotope & Reaction & CRONOS2 $(\mathrm{pcm} / \%)$ & IFP $(\mathrm{T} 4)(\mathrm{pcm} / \%)[6]$ & $\begin{array}{c}\text { Difference IFP } \\
\text { (T4)-CRONOS2 }(\%)\end{array}$ \\
\hline U234 & Capture + Fission & -1.38 & $-1.46 \pm 1.97 \mathrm{E}-03$ & -5.48 \\
U235 & Capture + Fission & 362.82 & $336.00 \pm 1.58 \mathrm{E}-01$ & 7.98 \\
U238 & Capture + Fission & -215.80 & $-174.00 \pm 6.12 \mathrm{E}-02$ & 24.02 \\
B10 & Capture & -163.91 & $-157.00 \pm 5.05 \mathrm{E}-02$ & 4.40 \\
\hline
\end{tabular}

Table 3. Uncertainty on reactivity due to nuclear data with CRONOS2 sensitivities at 4 energy groups.

\begin{tabular}{lrrr}
\hline Isotope & Capture & Fission & Total $(\mathrm{pcm})$ \\
\hline U235 & 133.69 & 152.47 & 202.78 \\
U238 & 264.90 & 357.30 & 444.79 \\
B10 & 76.66 & 0.00 & 76.66 \\
Zr90 & 10.30 & 0.00 & 10.30 \\
Zr91 & 41.75 & 0.00 & 41.75 \\
Zr92 & 35.34 & 0.00 & 35.34 \\
Zr94 & 2.72 & 0.00 & 2.72 \\
Zr96 & 6.95 & 0.00 & 6.95 \\
H2O & 188.00 & 0.00 & 188.00 \\
Total $(\mathrm{pcm})$ & 363.89 & 388.47 & 532.29 \\
\hline
\end{tabular}

they are not shown in this paper. IFP method has been developed for continuous energy Monte Carlo code to calculate adjoint flux by counting the expected fission neutrons produced in some future time after a neutron is introduced into the system. On the other hand, IFP calculations has also been done with Monte Carlo code TRIPOLI4 for the most sensitive isotopes. Comparison between CRONOS sensitivities and TRIPOLI4 IFP are in Table 2. The differences between these two calculation methods are small for the most sensitive isotopes (U235, $\mathrm{U} 238, \mathrm{H} 2 \mathrm{O}$ and B10) but is nearly of $20 \%$ for capture of U238. This gap is explained by employed calculation scheme and particularly by self-shielding which has an impact on the pertinence of sensitivities. Differences can be more important for less sensitive isotopes such as Zr90, Zr91, Zr96, Sn115, Sn117 and Sn118. Their sensitivities are so weak that these differences will not have consequence on uncertainties calculations. Finally, sensitivities calculations by SPT in CRONOS2 code is validated. In addition, sensitivities calculations with CRONOS2 is compatible with industrial constaints. In fact, calculation time is nearly $1 \mathrm{~h}$ to obtain all these sensitivites for only one CRONOS2 calculation.

\section{Reactivity uncertainty due to nuclear data of BEAVRS benchmark}

CRONOS2 sensitivities calculated in Section 5 are combined with four energy groups covariance matrix to estimate uncertainty on reactivity due to nuclear data according to the following equation:

$$
\varepsilon(k, \sigma)=\sqrt{S^{t} M S} .
$$

In this equation, $S$ is the sensitivities vector containing sensitivity of parameter $k$ to cross section $\sigma$ in each energy group. And $M$ is the corresponding covariances matrix. These matrix result from condensation of COMAC matrix at 49 energy groups (COMAC V1.0) [13-15]. Condensation method relies on the preservation of total variance for a given isotope. For this, sensitivities are calculated with APOLLO2 code with 49 energy groups and coupled with covariance matrix. Results by isotope and reaction are given in Table 3 . The total uncertainty on reactivity is $532 \mathrm{pcm}$ for ARO configuration. It value is close to that calculated in references $[16,17]$. The greatest contributor to uncertainty is U238 which represents nearly $50 \%$ of the uncertainty on the reactivity.

\section{Conclusion}

This paper proposed a calculation method for reactivity sensitivities due to nuclear data at industrial scale for a 3D modeled core. Unlike Monte Carlo methods which are expensive and time-consuming, the developed CRONOS2 procedure takes advantage of using a short calculation time. It is based on SPT. Consequently, it is able to calculate sensitivities for all interest isotopes and for all reactions (except scattering reaction) and energy group with a short time calculation (nearly $1 \mathrm{~h}$ ). CRONOS2 procedure has been validated comparing calculated sensitivities to IFP results. Differences between these two calculations methods are small, especially for the most sensitive isotopes such as U235, U238 and B10 but capture of U238 presents a gap of nearly $20 \%$. To reduce this gap, this development requires to be tested with other calculation scheme to study the impact of self-shielding choice or the creation of equivalent coefficients which would preserve reaction rate coupled with adjoint flux These sensitivities have enabled to estimate uncertainty on reactivity due to nuclear data for the BEAVRS benchmark in ARO configuration. This uncertainty agrees with uncertainty calculated by other authors. To summarize this work, this study shows the capacity of CRONOS2 code to compute sensitivities and uncertainties in diffusion with only 4 energy groups. For the user, sensitivities calculation comes down to a simple procedure to obtain all reactivity sensitivities with only one calculation. For the qualification, this development enables to have quickly an estimation of reactivity uncertainties. Other configurations 
of BEAVRS core can be studied such as those which have control rods but they are more difficult to model.

The author wishes to thank G. Prulhiere for the development of BEAVRS scheme and C. Magnaud for her help on the implementation of sensitivities calculation in CRONOS2 code.

\section{References}

1. D. Rochman et al., Nuclear data uncertainty propagation: total Monte Carlo vs. covariances, J. Korean Phys. Soc. 59, 1236 (2011)

2. A. Santamarina et al., Validation of the new code package APOLLO2.8 for accurate PWR neutronics calculations, in International Conference on Mathematics and Computational Methods Applied to Nuclear Science and Engineering (SunValley, CD-ROM, 2013)

3. J.J. Lautard et al., CRONOS a modular computational system for neutronic core calculations, in Advanced Calculational Methods for Power Reactors (Cadarache, 1990), pp. $42-50$

4. MIT Computational Reactor Physics Group, Benchmark for Evaluation And Validation of Reactor Simulations, Release rev 2.0.1, 2017

5. L.N. Usachev, in Perturbation Theory for the Breeding Factor and Other Ratios of Different Processes in a Reactor (Atomnaia Energiya, 1963), p. 472

6. M.L. Williams, Perturbation Theory for Nuclear Reactor Analysis (CRC Handbook of nuclear reactor calculations, 1986)

7. J. Lewins, Importance, the Adjoint Function (Pergamon Press, 1965)
8. G. Truchet et al., Computing adjoint-weighted kinetics parameters in TRIPOLI4 by the Iterated Fission Probability method, Ann. Nucl. Energy 85, 17 (2015)

9. G. Truchet et al., Implementation and validation of reference sensitivity profile calculations in TRIPOLI4, in ICNC (2015)

10. Y. Qiu et al., Computing eigenvalue sensitivity coefficients to nuclear data based on the CLUTCH method with RMC code, Ann. Nucl. Energy 88, 237 (2016)

11. A. Santamarina et al., The JEFF3.1.1 Nuclear Data Library - JEFF Report 22: validation results from JEF-2.2 to JEFF3.1.1, AEN data bank, 2009

12. N. Hfaiedh et al., Determination of the optimised SHEM mesh for neutron transport calculations, in Mathematics and Computation (2005)

13. C. De Saint Jean et al., Uncertainty evaluation of nuclear reaction model parameters using integral and microscopic measurements with the CONRAD code, J. Korean Phys. Soc. 59, 1276 (2011)

14. C. De Saint Jean et al., Estimation of multi-group cross section covariances of $238,235 \mathrm{U}, 239 \mathrm{Pu}, 241 \mathrm{Am}, 56 \mathrm{Fe}$ and $23 \mathrm{Na}$, in PHYSOR (2012)

15. E. Privas et al., Generation of U238 covariances matrices by using the integral data assimilation technique of the CONRAD code, in EPJ Web of Conferences 106 (2016)

16. C. Wan et al., Uncertainty quantification of the BEAVRS benchmark problem at the steady state, in International Conference on Mathematics and Computational Methods Applied to Nuclear Science and Engineering (2017)

17. C. Wan et al., Uncertainty analysis for the assembly and core simulation of BEAVRS at the HZP conditions, Nucl. Eng. Des. 315, 2011 (2017)

Cite this article as: Julien Gaillet, Thomas Bonaccorsi, Gilles Noguere, Guillaume Truchet, Development and validation of uncertainty neutron transport calculations at an industrial scale, EPJ Nuclear Sci. Technol. 4, 45 (2018) 